Cahiers Charlevoix

Études franco-ontariennes
Cahiers Charlevoix Études franco-ontariennes

or Clevenerite of:

\title{
Jean Éthier-Blais. Interview (1989)
}

\section{Michel Gaulin}

Volume 4, 2000

URI : https://id.erudit.org/iderudit/1039362ar

DOI : https://doi.org/10.7202/1039362ar

Aller au sommaire du numéro

Éditeur(s)

Société Charlevoix

Presses de l'Université d'Ottawa

ISSN

1203-4371 (imprimé)

2371-6878 (numérique)

Découvrir la revue

Citer ce document

Gaulin, M. (2000). Jean Éthier-Blais. Interview (1989). Cahiers Charlevoix, 4 ,

301-340. https://doi.org/10.7202/1039362ar d'utilisation que vous pouvez consulter en ligne.

https://apropos.erudit.org/fr/usagers/politique-dutilisation/ 


\title{
DocumenT
}

\author{
JeAn ÉthieR-Blais \\ INTERVIEW (1989)
}

Propos recueillis par Michel Gaulin

Cahiers Charlevoix 4, 2000, pp. 301-340. 


\section{SOMMAIRE}

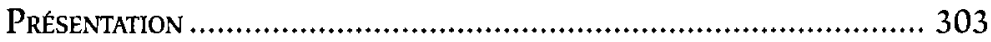

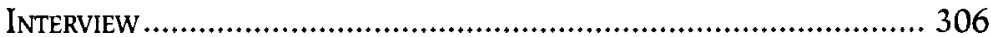

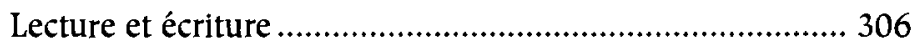

Une "petite ville perdue du nord de l'Ontario»: microcosme de culture ................................................ 308

Sudbury: le collège, l'amitié, la lecture ............................. 315

L'écriture ................................................................... 322

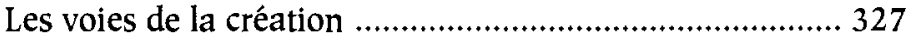

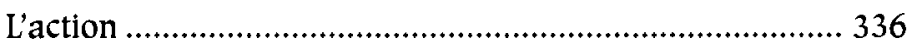




\section{JEAN ÉTHIER-BLAIS \\ INTERVIEW (1989)}

Propos recueillis par Michel Gaulin

\section{Présentation}

Nous reproduisons ci-après le texte intégral d'une interview que nous accordait, chez lui, au 3425 , avenue Ridgewood, à Montréal, le jeudi 16 mars 1989, Jean Éthier-Blais. En prévision du congrès international du PEN Club ${ }^{1}$ qui devait se tenir à Toronto et à Montréal, du 23 au 29 septembre 1989 et pour lequel Éthier-Blais, en tant que président du Centre francophone canadien (bientôt d'ailleurs rebaptisé, sur son initiative, "québécois») du PEN, assumait la responsabilité du volet montréalais, le directeur fondateur de la revue Lettres québécoises, Adrien Thério, avait eu l'heureuse idée de lui faire l'honneur du numéro d'été 1989 de sa revue. Ainsi, sa photo paraîtrait en page couverture et l'interview qui constitue, dans chaque livraison, l'article vedette lui serait consacrée. Thério eut l'amabilité de me confier le soin de cet entretien. En raison, toutefois, des contraintes d'espace inhérentes à ce genre d'initiative, c'est une version fort abrégée

\footnotetext{
${ }^{1}$ Le PEN Club (Poets, Playwrights, Editors, Essayists, and Novelists) est un regroupement international d'écrivains, dont le siège est à Londres, et qui se donne pour mission l'entraide entre écrivains et, particulièrement, l'aide aux écrivains en prison.
} 
(et, par conséquent, en partie remaniée) du texte d'origine qui parut dans la revue ${ }^{2}$.

Il nous a semblé que la qualité des propos tenus par Éthier-Blais à cette occasion justifiait la publication du texte en son entier, et d'autant plus que de nombreux éléments en recoupent ou appuient l'étude que nous consacrons, ailleurs dans le présent numéro des Cahiers, à l'entreprise mémorielle que constituent Fragments d'une enfance $e^{3}$ et Le Seuil des vingt ans $\mathrm{s}^{4}$. Au printemps de 1989, Éthier-Blais venait d'achever la rédaction du premier de ces deux ouvrages. Il avait donc encore très présents à l'esprit les motifs qui forment la trame de ce premier tome de souvenirs, et songeait déjà à ceux dont il entendait faire usage dans le second tome, appelé à paraître trois ans plus tard.

C'est sans doute ce qui explique que nous retrouvions des traces si abondantes de ces divers motifs dans les parties de l'entrevue qui traitent de sa ville natale, Sturgeon Falls, des paysages si caractéristiques du nord de l'Ontario, de même que de ses études chez les jésuites, à Sudbury. Comme Éthier-Blais y fait lui-même allusion en cours d'entretien, ces propos constituent un témoignage précieux sur la vie de la communauté française du nord de l'Ontario au cours des années trente et de la première moitié des années quarante du siècle qui s'achève. En même temps, l'entrevue nous paraît jeter une lumière éclairante sur la façon dont s'est développée, en Ontario français, sa vocation d'écrivain et sur la manière dont, parvenu aux dernières années de sa vie, il concevait son métier qu'il continua à exercer activement jusqu'à ce que la mort vînt trop tôt y mettre un terme.

$\mathrm{Si}$, par ailleurs, les propos qu'il tient, en fin d'entrevue, sur les préparatifs du congrès international du PEN Club

\footnotetext{
2 "Jean Éthier-Blais: une vie en écriture", interview de Michel Gaulin, Lettres québécoises (Montréal), nº 54 (été 1989), p.10-13.

${ }^{3}$ Jean Éthier-Blais, Fragments d'une enfance, Montréal, Leméac, 1989.

${ }^{4}$ Jean Éthier-Blais, Le Seuil des vingt ans, Montréal, Leméac, 1992.
} 
dans lesquels il était alors engagé peuvent aujourd'hui, à quelque onze années de distance, paraître un peu datés par rapport à la valeur d'actualité qu'ils revêtaient en 1989, ils n'en montrent pas moins un côté de sa personnalité et de son tempérament que peu de lecteurs connaissaient à l'époque et qui, parallèlement à ses occupations d'universitaire et d'écrivain, l'inclinait à l'action. C'est pourquoi nous avons choisi de les conserver ici comme partie intégrante d'un témoignage reposant sur trois assises principales, soit:l'enfance et la jeunesse en milieu franco-ontarien; le métier d'écrivain; l'action.

Nous avons veillé, dans la transcription, à respecter dans toute la mesure du possible, l'intégrité des propos confiés par Éthier-Blais à notre magnétophone et leur allure de conversation. Ainsi, par exemple, nous avons conservé les points de suspension là où l'interviewé hésite ou ne mène pas sa phrase à terme parce qu'il saisit au vol une nouvelle idée qui vient de surgir dans son esprit. En revanche, nous avons, ici et là, débarrassé la transcription de certaines scories du texte parlé, comme beaucoup de ces "eh bien» et «naturellement», dont Éthier-Blais, comme tout locuteur, avait tendance, dans la conversation, à se servir comme de chevilles à l'expression de sa pensée, et qu'il n'aurait vraisemblablement pas voulu conserver lui-même si l'occasion lui avait été donnée de revoir son texte. Enfin, dans le but de faciliter la lecture et de guider le lecteur à travers un témoignage passablement long et dense (l'interview devait durer quelque deux heures et demie), nous avons inséré, entre ce qui nous paraissait être les principales articulations de l'entretien, des sous-titres de notre cru.

L'entrevue, on le constatera, commence, pour ainsi dire, in medias res, par un échange sur les rapports réciproques entre la lecture et l'écriture, comme si intervieweur et interviewé poursuivaient un dialogue déjà amorcé, alors qu'en réalité cet échange constitue bien le véritable point de départ de l'entretien. 
Nous tenons à remercier, en terminant, le directeur actuel de Lettres québécoises, monsieur André Vanasse, de même que le président directeur général de XYZ éditeur, monsieur Gaëtan Lévesque, de nous avoir aimablement autorisé à reproduire le texte intégral de cette entrevue réalisée sous l'égide de la revue.

Michel Gaulin

\section{INTERVIEW}

LECTURE ET ÉCRITURE

M. G. Jean Éthier-Blais, votre œuvre est à mon avis profondément marquée par le dialogue entre la lecture et l'écriture, l'une nourrissant l'autre. Est-ce aussi votre avis? Quelle sorte de lecteur êtes-vous, et comment l'exercice de la lecture influe-t-il sur votre écriture?

J. É.-B. Je n'ai jamais séparé la lecture du plaisir de la lecture. C'est-à-dire que quand j'ai commencé à lire j'étais très jeune, j'avais peut-être quatre ans, peut-être cinq ans, j'oublie, mais enfin j'étais petit garçon - j'ai lu des choses par plaisir. Pour moi, c'était un plaisir. Si je n'avais pas eu ce plaisir de la lecture, eh bien je crois, naturellement, que je n'aurais pas lu. Et ce plaisir est devenu un plaisir non pas solitaire, parce que cela c'est très général - la lecture est un plaisir solitaire - mais est devenu un plaisir d'autant plus agréable que la lecture m'amenait à me retrancher de mon camp familial. C'està-dire que je prenais mon livre et je partais, j'allais m'installer dans un grenier ou dans une chambre, fermée à clef de préférence, où personne n'entrait, et là je pouvais me livrer pendant des heures au plaisir de lire et au plaisir de l'imagination. Et mon imagination remplaçait les images. J'ai peu lu d'ouvrages illustrés, même dans mon enfance. Le texte lui-même, les lignes qui se suivaient sur la page, cette admirable composition du texte qui devient, en soi, une image, une image qui bouge comme dans les paravents 
chinois, où on déroule le paravent et l'image continue on voit toute la trajectoire du récit sur le paravent à mesure qu'on le déroule - eh bien, pour moi, enfant, les pages du livre représentaient ce déroulement de l'histoire.

J'ai toujours lu, enfant, à deux niveaux, au niveau de l'histoire qu'on me racontait, et au niveau de la présentation matérielle du texte, comme si le texte était illustré par des toiles non figuratives. Et je retrouve cela, aujourd'hui, même dans le décor dans lequel je vis, où il $y$ a beaucoup de toiles non-figuratives. Il y a une alternance de toiles figuratives, qui racontent l'histoire, donc qui sont anecdotiques, qui sont un texte, mais ces toiles figuratives sont presque toujours accompagnées de toiles non figuratives qui, elles, représentent l'abstraction, l'essence même de ce déroulement de toiles figuratives. Donc la lecture, pour moi, très tôt, s'est située à ce niveau, et c'est pourquoi les illustrations ne m'ont jamais vraiment intéressé. Parce que l'illustration, pour moi, dans un texte, c'était la présentation du texte lui-même. Et j'aimais, déjà, enfant, prendre un livre et le lire, c'est-à-dire tout simplement tourner les pages pour engranger des images abstraites en noir et blanc. Et je le fais encore aujourd'hui. Ainsi, je prends un livre, par exemple une Pléiade, et c'est pour moi un très grand plaisir de lire sans lire, c'est-à-dire de voir comment un auteur conçoit son texte par blocs - un auteur comme Stendhal, par exemple, qui écrit des petits paragraphes qui se suivent les uns les autres de façon très très nerveuse, ou un auteur, naturellement, comme Proust, qui lui, au contraire, remplit ses pages, inonde les pages de mots et de phrases, et de lignes en noir et blanc. Eh bien, pour moi, c'est aussi un critère de science d'écriture et de conception esthétique de l'écriture.

Alors, vous voyez, dès le début de ma lecture, dès que j'ai commencé à lire, j'ai lu à partir de certaines données. Et ces données reposaient essentiellement sur la notion de plaisir. Et peu à peu, à mesure que je vieillissais, à mesure 
que j'apprenais à réfléchir, j'en suis venu à la conclusion que la réflexion était elle-même un plaisir, ce qui fait que lorsque je lis un texte, par exemple de Schopenhauer pendant une heure je vais lire du Schopenhauer - , pour moi la réflexion de Schopenhauer, le mécanisme de construction d'une pensée représente un plaisir, comme le mécanisme du montage psychologique représente un plaisir. Alors, quand je lis, je lis toujours en fonction de cette découverte d'un plaisir nouveau. Le style, naturellement, est, lui, un renouvellement constant de ce plaisir, lorsque style il y a...

UNE «PETITE VILLE PERDUE DU NORD DE L'ONTARIO»: MICROCOSME DE CULTURE

M. G. Revenons en arrière, si vous le voulez bien. Vous disiez, tout à l'heure, que la lecture vous a d'abord permis, dans l'enfance, de vous abstraire un peu de votre camp familial. Nous savons, par vos livres, que votre enfance dans ce que vous avez appelé "cette petite ville perdue du nord de l'Ontario", a été très importante. Mais, en fin de compte, cette petite ville française du nord de l'Ontario, c'était aussi un milieu fermé, dont vous avez sans doute senti le besoin de vous évader. On en voit la trace, par exemple, dans certaines pages du Dictionnaire de moi-même - ce besoin, aussi, d'échapper à ce milieu fermé. Est-ce que, effectivement, le milieu de l'enfance était un milieu fermé et comment avez-vous ressenti ce besoin de vous en échapper?

I. É.-B. C'était un milieu fermé, mais au niveau de l'histoire. Il était fermé parce qu'on avait tout simplement rabattu le couvercle sur cette petite population française du nord de l'Ontario. Mais ce n'est pas elle qui avait rabattu le couvercle. Cette population, au contraire, avait un immense besoin de partir. Par exemple, dans ma rue, je vois maintenant, je revois les maisons de ma rue et les gens 
qui habitaient ces maisons. Eh bien, je constate que - il y avait peut-être vingt-quatre maisons dans cette rue, depuis le coin que nous habitions, jusqu'à l'église, rue Lévesque. De ces vingt-quatre maisons sont sorties, facilement, trente personnes qui ont fait des études secondaires et universitaires et qui ont quitté la ville et qui sont venues habiter Montréal, ou bien Ottawa, ou même Québec. Donc, ce besoin d'évasion, de quitter une ville qui ne pouvait pas répondre aux aspirations profondes et adultes de ses habitants, ce besoin existait pour tout le monde. Je ne suis pas une exception dans ce cas.

Mais j'ai vécu quand même ce besoin de fuite, une fuite en avant vers un milieu qui me permettrait - tout cela inconsciemment, je crois -, qui me permettrait de m'épanouir. Mais, par contre, ce milieu très fermé, comme tous les milieux fermés - comme les milieux de Mauriac ou de Julien Green -, ce milieu fermé permet aussi de vous approfondir. Alors, pour un enfant qui ne peut pas échapper au milieu, qui suit ses parents en voiture ou dans le train ils disent: «Bon, nous partons, nous allons à Montréal, nous allons à Pointe-Claire voir des parents» —eh bien, il monte dans la voiture et puis il suit. Alors il ne peut pas s'enfuir, même s'il veut s'enfuir, ou bien il s'enfuit uniquement par l'imagination. Mais sa fuite à lui, son moyen de fuite, c'est de s'installer à l'intérieur de lui-même et de creuser des tunnels dans son for intérieur. Et la lecture aide beaucoup, à ce moment-là, à créer ces tunnels et à créer toute une fourmilière à l'intérieur de soi-même, qu'on habite - on est toutes les fourmis en soi-même. C'est une fourmilière qui est très habitée, mais qui n'a en réalité qu'un seul habitant multiforme.

Donc, vous avez cet épanouissement intérieur chez l'enfant qui veut bien se donner cet épanouissement, ou alors qui y est porté par un inconscient très fort. D'une part, vous avez cet épanouissement intérieur et d'autre part, dans mon cas, il y a quand même des éléments très importants de cette 
nature du nord de l'Ontario, qui est représentée par les contreforts des Laurentides. Les Laurentides débutent là... ou elles se terminent, mais je pense qu'elles débutent dans le nord de l'Ontario. Vous avez un paysage, l'un des plus beaux paysages qui existent, à mon avis, dans le monde entier.

La première image qui m'a frappé, dans mon enfance, c'est l'image de la rivière. Et vous voyez, là, immédiatement, vous avez ce thème des grands départs, et de la fuite, et de la recherche du grand large. Parce que cette rivière, qui est la rivière de l'Esturgeon, elle a accompagné mon enfance. D'abord, $j$ 'ai un de mes frères qui est mort quand j'avais quatre ans - il en avait sept et il est mort noyé dans cette rivière. Déjà, c'est un élément très important au point de vue psychologique. C'est la présence de la mort de l'enfant. C'est moi, au fond, qui aurait pu mourir, parce que j'étais exactement comme lui, je courais avec des amis en direction de la rivière. Lui, il a plongé dans les chutes et il a été emporté et il est mort...

Alors, cette rivière qui représente le mouvement, et qui représente le mouvement nordique, en plus, cette rivière était entourée d'autres rivières. La rivière de l'Esturgeon était... elle avait des confluents, si vous voulez. Par exemple, une rivière qui allait de la rivière de l'Esturgeon jusqu'à une petite ville qui s'appelait Verner, où nous avions des amis - pour aller les voir, nous longions cette rivière. Et cette rivière portait le nom de la Veuve, ce qui est profondément français, c'est-à-dire cette rivière, c'est la Vouivre de Marcel Aymé, c'est la femme-serpent, c'est la veuve qui est à la recherche d'un second mari et qui attire, c'est tout le thème de Lorelei, en réalité, qui revient dans cette rivière de la Veuve. Il y a eu souvent des hommes qui voyageaient la nuit au bord de cette rivière en voiture et qui s'endormaient au volant, qui perdaient le contrôle du volant, qui descendaient doucement sur les berges de la rivière et qui disparaissaient dans la rivière. La Veuve, cette rivière avait 
une sorte d'aura magique dans mon enfance. Je dis: elle nous menait à Verner. Ce n'est pas vrai. C'était à Field qu'elle menait, aux champs, vers les champs lointains. Alors, vous aviez cette rivière qui se jetait dans la rivière de l'Esturgeon et la rivière de l'Esturgeon qui, juste avant d'arriver dans ma ville, s'élargit et devient presque un petit fleuve - elle est très douce, somnolente, elle descend très lentement et, tout d'un coup, en plein milieu de ma ville, il $y$ a des chutes formidables, deux grandes chutes, et cette rivière se transforme en chutes, elles aussi maléfiques (mon frère, par exemple, qui y meurt)...

J'ai passé toute mon enfance dans le bruit de ces chutes. Donc, la nature est très présente d'une façon évidente, immédiate dans mon cerveau. La première musique que j'ai entendue, c'est la musique d'une rivière et des chutes dans une ville, qui éclatent et qui parfois sont très bruyantes et qui parfois, naturellement, quand le cours d'eau est moins fort, la chute, elle aussi, est moins audible. Et aussitôt après les chutes, dans ces tourbillons extraordinaires que nous allions voir - les tourbillons des eaux, nous allions nous amuser, pendant des heures, à regarder les tourbillons d'eau dans la chute - immédiatement après, la rivière reprend un cours paisible et elle devient une sorte de delta, très beau d'ailleurs, et elle s'élargit, et elle descend très lentement vers le lac Nipissingue.

Ce lac aussi a joué un grand rôle dans ma vie d'enfant, parce que c'était le lieu des vacances, c'était la grande nappe d'eau immense qui représente une sorte de mer intérieure, mais c'était aussi un dieu maléfique parce que j'ai beaucoup d'amis de ma famille qui y sont morts noyés des jeunes, des jeunes gens, des jeunes filles, qui se noyaient dans ce lac, des soirs d'été. Il y avait donc la présence de cette eau très puissante qui jouait un rôle dans la vie des gens. C'est le premier élément d'échappée sur le cosmos, si vous voulez. 
Le deuxième, c'est le ciel. Je ne sais pas si vous connaissez ces ciels du nord de l'Ontario, qui sont très beaux et qui donnent cette impression d'immensité et de divinité. Dieu existe quand on voit ce ciel. Et alors pour moi, enfant, comme mon père est mort quand j'étais très jeune et qu'il était enterré dans le cimetière et que, pour aller au lac, nous devions passer à côté du cimetière, ce ciel extraordinaire, il est associé, pour moi, à l'idée d'une divinité. C'est un ciel très bas, un ciel qui n'en finit plus, un ciel qui n'est pas très coloré. Mais il est présent -c'est encore l'idée du couvercle, si vous voulez - il est présent, il termine, il met le point final à la réalité physique de la ville, à la réalité cosmique. Ce ciel menait directement au lac. Il y avait là le cimetière, à côté, avec mon père qui repose dans ce cimetière, maintenant ma mère - pour moi, c'est une réalité extrêmement présente. Je ne vais jamais dans ma ville natale sans aller m'incliner sur la tombe de mes parents, et souvent je vais dans ma ville natale uniquement pour m'incliner sur la tombe de mes parents, parce que je crois très profondément à l'existence éternelle de ce lien, de cette filiation, de ce rapport entre un homme et une femme qui ont créé un enfant, qui est devenu un homme, cet homme qui redevient un enfant lorsqu'il est devant la tombe de ses parents.

Alors vous voyez, donc, qu'il y a une sorte d'approfondissement intérieur par la lecture et une échappée que je peux appeler véritablement cosmique - à mon niveau, naturellement - mais [qu'il y a] ce sens de l'appartenance et de l'unité dans la réalité physique de la terre et du ciel dans toutes ces manifestations presque anthropomorphiques de l'univers qui nous entoure.

M. G. Donc, enfance en milieu fermé, mais c'est peutêtre ce genre d'enfance, précisément, qui vous a permis de développer très tôt les puissances de votre imagination, que vous n'auriez pas pu développer autrement. 


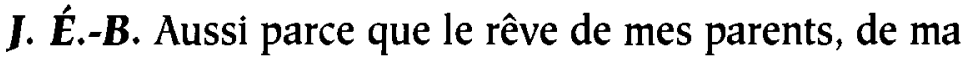
mère en particulier, parce que mon père est mort j'étais jeune (mais il aurait été enchanté lui aussi), leur rêve c'était d'avoir un de leurs enfants écrivain. Et je me rends compte maintenant que si j'ai autant tardé à écrire, c'est parce que je ne voulais pas faire ce plaisir à mes parents. Ils attendaient un écrivain dans la famille. Mes deux frères n'ont pas choisi cette voie, mais moi je l'ai choisie parce que ma famille était une famille littéraire, pas nécessairement parce que tout le monde y lisait beaucoup, pas parce qu'ils étaient au courant des choses de la littérature ou qu'ils avaient fait des études littéraires approfondies. J'ai vécu dans une maison où il y avait énormément de livres, et toujours de plus en plus - on achetait constamment des livres. Mes frères faisaient des études à l'Université d'Ottawa et, naturellement, ils en rapportaient des livres. On lisait aussi à cette époque; on n'a pas commencé à lire seulement en 1980. Mes sœurs faisaient des études, elles, au couvent de la rue Rideau, toujours à Ottawa. Elles aussi, elles en rapportaient des livres. Elles rapportaient des prix, elles rapportaient des livres qu'on leur avait fait acheter. Nous avions une bibliothèque...

Mes parents avaient constitué une bibliothèque pour leurs enfants. Par exemple - je donne des exemples parce que c'est intéressant au point de vue sociologique -, nous recevions la Revue des deux mondes - c'est très intéressant la Revue des deux mondes -, nous recevions $\mathrm{La} \mathrm{Se}$ maine de Suzette, aussi très intéressant. Nous avions tous les livres de la comtesse de Ségur, l'Encyclopédie de la jeunesse - je donne ces titres parce que cet Ontario français des années $1930 .$. Nous avions toute la collection Nelson, qui est très belle et très complète. J'ai lu des mémoires de généraux des guerres napoléoniennes quand j'avais dix ans, douze ans. Pour moi, c'était cela l'épopée, c'était cela Tintin. Tintin, c'était le général Marbot, et j'espère qu'il y $\mathrm{a}$ une différence entre les deux... Nous avions 
aussi toutes les grandes collections de chez Hachette, de livres d'aventures françaises en Algérie, au Tonkin. Je lisais tout cela. Et puis une grand série, qui m'a beaucoup intéressé, sur les contes et les traditions et les mythologies de toutes les civilisations. Et aussi des ouvrages de géographie, etc. Nous avions énormément de livres. Je vais chez une de mes sœurs, maintenant, qui a hérité de cette bibliothèque - c'est elle qui a tout pris, qui a tout raflé -, je vois les choses qui s'y trouvent et, même là, je les prends et je lis, et je trouve cela très intéressant. Ce n'étaient pas des livres véritablement d'enfants, c'étaient des livres, déjà, d'adultes. J'ai lu très tôt, d'abord des choses d'enfant, naturellement - est-ce que la comtesse de Ségur est un auteur d'enfants? je me le demande - et une très belle édition de La Fontaine - je me souviens de cela - un très beau livre relié, avec des photos, de ces photos anciennes, françaises, qui sont très mauvaises et tellement belles en même temps, qui sont à moitié brouillées, [un livre] qui s'appelait Les Merveilles de la France. Alors là, j'étais en pleine géographie, mais géographie racontée sur le mode historique. Le milieu dans lequel je baignais était un milieu littéraire.

Autour de moi, dans les maisons autour, les jeunes gens et les jeunes filles faisaient des études. Ils faisaient du piano... C'était un microcosme, cette petite ville. Je m'en rends compte maintenant, en réalité. Par exemple, en face de chez moi, mes meilleurs amis... Jacqueline Paiement: elle a eu un premier prix de piano du Conservatoire de Montréal et de [celui de] San Francisco. Ensuite, elle a décidé d'abandonner le piano, elle a fait de la médecine, elle est devenue psychiatre - je l'ai retrouvée comme collègue à McGill, où elle était psychiatre des étudiants. Elle est morte, hélas, elle avait peut-être cinquante-cinq ans. Son frère Raymond, qui était mon meilleur ami, est devenu lui aussi médecin, il allait... Vous comprenez, il y avait un va-et-vient de gens et de personnes, un échange d'idées 
(parce que tout le monde ne faisait pas ses études au même endroit), il y avait un grand choc d'idées dans cette petite ville, dans ce milieu bourgeois de la petite ville. Il y avait, comme dans toutes ces petites villes canadiennes, la voie ferrée qui séparait la ville en deux. Les gens qui habitaient de l'autre côté de la voie ferrée, naturellement, eux étaient presque personce non gratce. Mais, de mon côté à moi, c'était très bien, c'était ce milieu de culture. Mais vous me direz peut-être: c'était une culture quand même assez élémentaire. Ce n'était pas la culture du milieu de Proust, ou du milieu de Léautaud, mais c'était quand même un univers de culture, et c'est ce qui compte. Ce n'est pas le niveau qui compte, surtout à cet âge. C'est d'apprendre très tôt à respecter l'univers de la culture et à vivre dans un univers de culture et à ne pas pouvoir vivre, surtout, hors d'un univers de culture. C'est ce qui fait que, dans les camps de concentration, avec rien du tout, Messiaen écrit son Quatuor pour la fin du temps, avec des instruments qui n'ont rien à voir entre eux.

M. G. Mais, en fin de compte, après cette enfance qui, d'après le récit que vous en faites, semble avoir été une enfance paradisiaque...

I. É. - B. ... presque paradisiaque...

SUDBURY: LE COLLĖGE, L'AMITIÉ, LA LECTURE

M. G. ... Vous êtes tout de même parti. Vous êtes parti physiquement, d'abord vers Sudbury, puis ensuite vers Montréal, puis vers l'Europe. Mais vous êtes sans doute parti aussi intellectuellement. Et cela a dû contribuer à faire de vous l'écrivain que vous êtes devenu. Est-ce que vous voulez nous parler de ce qu'a représenté ce départ?

J. É.-B. Oui, mais je ne sais pas comment... Parce qu'il y a sûrement une césure à partir du moment où je suis entré au Collège des jésuites. D'abord, j'ai gagné ce prix, ce concours de français. Déjà c'est très significatif, que j'aie 
gagné ce prix provincial comme étant le petit garçon j'avais onze ans; j'ai eu douze ans en novembre ${ }^{5}$ - le petit garçon qui est le meilleur en français. Et surtout à cette époque, où cela avait une valeur d'exemple. C'était très symbolique pour l'Ontario. Déjà je portais le drapeau, en quelque sorte. J'étais le porteur des couleurs du français. $\mathrm{Et}$, très curieusement, dès que j'ai appris qu'il y avait ce concours et ce prix d'éducation au bout, eh bien, j'ai su que j'allais gagner - je raconte cela, d'ailleurs, dans mes premiers récits de souvenirs que je viens d'écrire. Il y a eu une sorte de certitude prémonitoire, que j'étais fait pour entrer dans le monde de la langue française. C'est très curieux... Je suis donc allé à Ottawa pour toutes les épreuves, etc. C'était assez dur, d'ailleurs. C'était très sérieux, ce concours. Il y avait des tas et des tas de candidats. Et dès la fin de la première matinée, je sentais, autour de moi, les gens qui avaient décidé - même mes concurrents -; c'était certain, le truc. Tout en n'étant pas certain, parce que, malgré tout, je venais de cette toute petite ville. Et vousmême qui êtes d'Ottawa, vous connaissez l'importance, làbas, du système d'éducation, etc. Ces gens étaient beaucoup mieux préparés que nous, que moi, en tout cas, quoique j'aie été très bien préparé par les Filles de la Sagesse.

Mais c'était ridicule, un peu, tout ce qu'on m'avait fait apprendre... c'était provincial, voilà. Cela a été ma première rencontre avec un milieu qui n'était pas provincial. Ç'a été cette rencontre à Ottawa. Je me sentais dans un milieu qui avait une résonance autre que celle de mon petit milieu à moi. Mais, malgré tout, j'ai gagné, pas parce que j'étais le meilleur, je crois. J'ai gagné parce qu'il fallait que je gagne. C'était écrit qu'il fallait que je gagne pour pouvoir accéder au domaine de la littérature. J'avais été choisi. Je crois que

\footnotetext{
${ }^{5}$ Erreur quant à la chronologie. C'est au printemps de 1938 qu'Éthier-Blais, né le 15 novembre 1925, remporte la palme du concours provincial de français. Il avait donc douze ans à ce moment-là, non onze, comme il l'affirme ici. Il aura treize ans en novembre.
} 
c'est le cas de tous les écrivains, d'ailleurs. À tous les niveaux, l'écrivain est choisi. Et lorsqu'il ne rend pas justice à ce choix, c'est à ce moment que sa vie est ratée. Il peut écrire de très mauvais livres, il peut écrire des livres sans intérêt, ou bien il peut écrire des chefs-d'œuvre, c'est exactement la même chose, sub specie aternitatis. C'est la même chose, mais il faut que l'écrivain rende justice au talent qui lui a été donné.

Alors donc, il y a eu, au départ, cette acceptation d'un choix. Je me souviens très bien que quand on a prononcé mon nom - on m'a appelé en me disant: "Vous avez gagné le prix» -, à ce moment-là, j'ai senti comme quelque chose de très lourd qui me tombait sur les épaules. Il y avait là une sorte de responsabilité devant mon instrument de travail, devant mon outil d'expression.

Et [cette responsabilité] je ne l'ai pas acceptée. Je me suis laissé beaucoup emporter pendant mes années de collège, à Sudbury, par une alimentation... - je me suis littéralement empiffré de lectures et d'éléments culturels, mais d'une façon assez peu systématique. Mais en étant très ouvert, comme une pâte qu'on va travailler. Alors je me suis remis, comme cela, entre les mains de ces jésuites et des amis que j'avais autour de moi. Je me suis remis, surtout, entre les mains des professeurs. Hertel est venu, naturellement. Il a eu une grande influence sur moi, à titre d'ami, pas tellement au point de vue intellectuel, J'ai rencontré des jésuites qui étaient des hommes infiniment plus cultivés que Hertel et qui ont eu beaucoup d'influence sur moi. Ils m'ont expliqué des textes. Vous savez, à seize ans, je savais par cour tous les poèmes de Mallarmé. C'est vous dire, à peu près, ce qu'on nous enseignait. Je lisais, chez les modernes, à peu près tout, depuis Le Disciple de Paul Bourget jusqu'aux pièces de Claudel et un poète que les jésuites, à cette époque-là, adoraient et qui était d'origine chilienne - mais il écrit en français, vous le connaissez, le nom me reviendra tout à l'heure [Neruda?]. 
On nous faisait lire tout, vous comprenez. Et en plus, quand on a vu que j'avais cette sorte de goût assez démentiel de la lecture, et que je retenais tout en même temps tout cela s'en allait dans le grand moulin qui battait tout le temps, et dont les ailes étaient toujours au vent -, eh bien les jésuites m'ont tout simplement ouvert la bibliothèque des Pères, où il y avait les véritables livres. Et c'est là [qu'à partir de] dix-sept ans, pendant trois ans, j'ai lu les Mémoires de Chateaubriand, j'ai lu Tallemant des Réaux - vous vous rendez compte, c'est assez extraordinaire... J'ai lu Les Trois Âges de la vie intérieure du père Garrigou-Lagrange je donne des titres, comme cela, pour montrer la diversité de tout cela -, Sainte-Beuve, naturellement...

Il y a eu, chez les jésuites, trois choses qui ont été importantes pour moi. Il y a eu [d'abord] les études. J'ai appris beaucoup de choses dans ce collège. Les cours étaient très forts, les professeurs étaient savants. Le professeur de philosophie, le père Dupuis, était un homme tout à fait remarquable - il nous apprenait à penser, à réfléchir, à organiser un texte, etc. C'était beaucoup plus cela, son cours, qu'un cours de doctrine, parce que la doctrine, c'était le thomisme classique. Mais, à partir de cela, il nous apprenait à nous former l'esprit. Alors, il y a tout le côté connaissances qui est important dans cette éducation.

Le deuxième élément qui, à mon avis, a été important, c'est l'élément de l'amitié, de la fraternité. Je me suis fait là des amis, en particulier Guy Lafond, le poète, Robert Vigneault, qui est maintenant professeur à l'Université d'Ottawa, et Raymond Tremblay, qui est prêtre dans le diocèse de Sudbury [Sault-Sainte-Marie]. Nous étions des inséparables. Je peux le dire en toute honnêteté - et je le dis avec d'autant plus de simplicité que moi j'ai écrit beaucoup de livres -, j'étais le moins bon des quatre. Mais, c'est curieux la vie. Ces gens qui avaient des talents extraordinaires, il leur manquait peut-être tout simplement cette chiquenaude initiale, ou ce don de l'ambition - qui est 
quand même une chose qui existe - de s'exprimer, de s'affirmer dans une réalité qui a un volet éternel. Ils étaient plus forts que moi, plus savants, ils avaient fait des lectures plus approfondies - un homme comme Tremblay, par exemple, était une sorte de génie au point de vue intellectuel -, mais ils avaient une modestie que moi je n'ai pas parce que je suis un écrivain. Si j'avais la modestie, je ne serais pas écrivain, et à partir du moment où je suis écrivain, je n'ai pas cette modestie. L'écrivain est aussi porteur d'une faille morale. Il lui faut l'attention. Il écrit aussi pour briser, [surmonter] des obstacles, vaincre. Il y a cela, aussi, qui existe chez l'écrivain. Et moi je l'avais sans le savoir ou, le sachant, en refusant d'exploiter cette réalité. C'est donc le deuxième volet, le volet de l'amitié, qui a remplacé chez moi toute forme d'amour.

Je peux dire que je suis quelqu'un qui n'a jamais aimé. C'est assez extraordinaire, quand on y pense. J'ai aimé mes parents, bien sûr, j'ai aimé des amis - non, pas des amis; je ne les aime pas; j'ai de l'amitié, c'est autre chose -, mais je n'ai jamais aimé, emporté par la passion et le désir. Je n'ai jamais désiré. Même maintenant je ne désire rien. Je ne veux pas dire physiquement, uniquement. Je n'ai jamais été amoureux d'une table, ni d'un tableau, ni d'un décor, ni d'une nature, ni d'un bateau sur la mer, ni d'un monument, de rien. Je suis absolument stérile à cet égard. Jamais je n'ai aimé, et je pense que je n'aimerai jamais. Je n'ai jamais eu... mon cœur n'a jamais battu plus vite, dans quelque circonstance que ce soit. Je suis un Bourbon. Les Bourbons étaient comme cela. Ils avaient cette froideur devant la réalité. Je peux me laisser emporter, mais c'est purement... cela relève du jeu, parce que je connais le maniement des processus sociaux. Je sais quand il faut que je me fâche, mais dans mon for intérieur je m'en contrefiche comme de ma première chaussette. Cela ne me touche absolument pas. Rien ne me touche. Sauf, peutêtre, le seul moment de passion que je puisse ressentir, 
c'est devant la musique, et de moins en moins. C'est vous dire le détachement dans lequel je vis. C'est assez terrible. Mais ce que je ressens profondément, $c$ 'est le sentiment de l'amitié et de la fidélité amicale. Cela je le ressens vivement et, pour moi, c'est la norme de l'affectivité dans mon cas. Je n'en ai pas d'autre. Et je ne comprends pas que l'on puisse être passionné. J'écris des livres où il y a des gens très passionnés qui se tuent, et qui assassinent, et c'est de la pure imagination, ou alors de la pure constatation objective d'une réalité qui nous entoure. Et je pense que je le fais avec d'autant plus de passion que je ne la ressens pas, cette passion. Vous savez vous-même qu'un homme très amoureux, par exemple (et je garde des lettres complètement idiotes - «je t'aime, je t'aime») ... mais Stendhal qui, en réalité, n'a jamais aimé - il a eu des crises de passion imaginative; il changeait même le nom des femmes qu'il aimait; c'est dire qu'il ne les aimait pas beaucoup; il leur donnait un autre nom, comme à une bonne mais, précisément parce qu'il y avait chez lui cet élément de froideur objective, il pouvait décrire cette réalité. Mais il faut accepter tout cela. Stendhal l'a accepté quand il avait vingt ans. Moi je l'ai accepté quand j'en avais cinquante. J'ai trente ans de retard sur lui.

Le troisième élément, naturellement - parce que tout se fait par trois - le troisième élément, ce sont ces lectures, c'est le système de lectures qui avait été mis en place, à Sudbury, chez les jésuites, pour certains étudiants triés sur le volet. C'était une introduction à la lecture universelle. Je disais à Gérard-Marie Boivin ${ }^{6}$ - vous avez entendu — Rilke, mais Rilke c'est un de ces écrivains qu'on nous faisait lire. Dostoïevski, naturellement, était un dieu pour nous, et nous lisions tout Dostoïevski, le Journal d'un écrivain, Les Frères

\footnotetext{
${ }^{6}$ Gérard-Marie Boivin, journaliste et animateur culturel qui, quelques semaines avant la présente entrevue, avait lui-même interviewé Éthier-Blais pour une station radiophonique culturelle de Montréal. Éthier-Blais et moi avions écouté un enregistrement de cette interview avant d'entreprendre la nôtre.
} 
Karamazov. La Bible était un point d'ancrage, aussi. Mais pas la Bible parce que c'est un document religieux. La Bible parce que c'est un chef-d'œuvre de la littérature universelle. Il y avait donc toute cette littérature, de Gerhardt Hauptmann... enfin on lisait beaucoup, toutes sortes de choses traduites, dans la littérature allemande (c'est là que j'ai commencé à suivre des cours d'allemand, à apprendre l'allemand, c'est chez les jésuites, à Sudbury - tout cela en pleine guerre, naturellement). On donnait des cours non pas d'introduction à la littérature, mais des cours de littérature par la lecture. Ce qui fait que, très, très tôt, j'ai eu cet esprit cosmopolite dans le domaine littéraire et qu'aujourd'hui je lis à peu près autant d'ouvrages étrangers - souvent traduits en français, d'ailleurs - que d'ouvrages français. Et je lis, je dois dire, de moins en moins d'ouvrages français contemporains. Je ne connais pas du tout la littérature française contemporaine. Je me suis arrêté à Roger Martin du Gard, Marcel Aymé - ce sont à peu près pour moi les auteurs les plus contemporains. Julien Gracq est sans doute le dernier que je connaisse. Ou alors je vais lire des ouvrages d'ordre purement anecdotique, ou alors des ouvrages de très haute qualité historique des mémoires, des correspondances, des essais historiques. Cela, je lis volontiers, comme le Louis XIV de Bluche, ou le Frédéric de Kantorowicz... enfin je lis le plus de choses possible dans des domaines qui m'intéressent. Mais cela, c'est le vieillissement, que voulez-vous. Et les auteurs que je lis véritablement, en français - c'est idiot à dire, d'une certaine façon -, c'est La Fontaine, que j'appelle «mon bon maître" - quand je parle de La Fontaine, je l'appelle «mon bon maître» - et Saint-Simon, que j'appelle «mon cher dieu». Ce sont mes deux auteurs véritablement de chevet. Ensuite, je lis Racine, Pascal - vous voyez, c'est le retour à la véritable réalité littéraire - ou des mémoires de cette époque. Je m'éloigne de plus en plus de mon siècle, pour me retrouver dans mon siècle. 
L'ÉCRITURE

M. G. Mais ces années d'études à Sudbury, les années d'études à Paris, en Allemagne, les années de diplomatie, en ce qui a trait à l'écriture, ce sont, pourrait-on dire, des années au cours desquelles vous avez engrangé, emmagasiné mais, par rapport à l'écriture au sens strict, ce sont des années de latence. Je sais qu'en 1944 vous avez publié des poèmes dans Gants du ciel, mais on pourrait dire qu'à cette époque-là vous écriviez probablement par devers vousmême. L'écriture, pour vous, d'après ce que je connais de la chronologie de votre vie, est venue au début des années soixante. Est-il juste de dire que le déclenchement immédiat de la carrière d'écrivain, ç'a été votre entrée au Devoir, en 1961 ?

J. É.-B. Oui, parfaitement. Les poèmes que j'ai faits en 1944, vous savez, c'est Hertel qui m'a tout simplement dit: "Mon vieux, il me faut trois poèmes pour demain.» C'est tout. J'avais une telle facilité... C'est ce qui, d'ailleurs, m'a fait beaucoup de tort. C'est cette facilité. Même en France, tout jeune, les gens, quand j'écrivais des lettres à des amis, me disaient: "Mais pourquoi n'écrivez-vous pas?", parce que eux sentaient en lisant la lettre - il y avait sans doute une sorte de frémissement d'écriture -, ils devinaient l'écrivain. Et moi, je me refusais à cela; je ne voulais pas être écrivain. Pourquoi? Je ne sais pas. Peut-être, comme je vous le disais tout à l'heure, pour refuser ce plaisir à mes parents? Oui... Peut-être aussi parce que je sentais que ce serait une occupation permanente et que mon côté malgré tout - parce que nous avons tous cela - mon côté passif m'empêchait d'accepter cette réalité super active que j'ai maintenant - et je ne m'en porte pas plus mal, remarquez. Mais cela, les poèmes, Hertel m'a tout simplement dit: "Écoutez, mon vieux, il me faut trois poèmes de genre différent pour... Je veux que votre nom figure dans cette liste" et c'est tout. Alors, je me suis installé à ma table de travail - je vois encore la petite table, dans la salle d'étude 
des élèves, à Sudbury -, et puis j'en ai fait deux, un le matin, un l'après-midi, le troisième le soir. Dans des genres différents, en me mettant un peu dans l'atmosphère, et hop... un sonnet, un machin sentimental, un troisième un peu plus objectif - exactement ce qu'il voulait.

Donc, il y avait cette facilité. Et je n'ai jamais bien écrit que sur commande. Quand on me demande quelque chose et que je le fais, je le fais avec énormément de plaisir et j'aime écrire dans ces circonstances. Je n'aime pas me commander à moi-même un texte. C'est pourquoi quand j'écris un roman, c'est toute une équipée, c'est comme le capitaine Scott se rendant dans l'Antarctique, parce que je suis obligé de me commander à moi-même un texte. Alors je l'écris parce que je suis organisé. Je me dis: «Bon, maintenant, c'est le devoir qui l'emporte. Il le faut!» Sur mes manuscrits, dans les marges, il y a toujours ce machin Beethoven: "Muss es sein? Es muss sein.» J'écris très souvent cela dans les marges, parce que je me dis: «Le faut-il? Oui, il le faut. " Parce que c'est la réponse à ma nature qui me dit «il le faut». C'est mon impératif catégorique.

Alors, je suis entré au Devoir - je l'ai raconté tellement de fois, je ne vais pas le raconter de nouveau - tout à fait par hasard. Mais cela aussi c'était un appel pressant du destin. Mais le destin m'a donné, en même temps, une très dure leçon, parce qu'il m'a fait me développer dans un genre qui n'est pas le mien, c'est-à-dire la critique littéraire. Je ne suis pas un critique littéraire. Alors, c'est très ennuyeux...

Je suis un critique littéraire en ce sens que je peux lire un livre; j'ai la formation. Je lis un livre, je sais ce qu'il y a dedans, je peux parler de ce qu'on dit ou de ce que raconte l'auteur, et puis je peux porter un jugement sur une œuvre - j'ai beaucoup lu. Instinctivement, tout ce que j'ai lu se replace, les armées, les corps d'armées se mettent en branle, les généraux sont là, sabre au clair, et 
les colonels, les capitaines, tout le monde est en place. Quand je porte un jugement sur l'œuvre de Marie-Claire Blais, il y a Dostoïevski, il y a Tolstoï, il y a Montaigne, il $y$ a Racine, il y a des tas de gens qui entrent en ligne de compte dans ce fonds subconscient. Donc, je peux faire de la critique littéraire, et puis j'ai acquis avec le temps - j'en fais depuis vingt-huit ans - une sorte de maitrise de la technique. Je connais tous les trucs. Par exemple, j'écris tout cela à la machine. Eh bien, quand je suis rendu aux deux tiers de la troisième page - puisque j'en fais trois avant de commencer à taper la troisième page, je mets un petit trait un peu après le deuxième tiers et quand ce trait apparaît sur le chariot, là je sais que je dois commencer à penser à ma chute d'article. Vous voyez, c'est un truc. Mais tout est truc. Je sais que je dois commencer à penser à ma chute d'article et tout en rédigeant, la chute d'article commence à prendre corps, etc. Parfois elle est réussie, parfois elle ne l'est pas. Mais la technique de l'écriture est très développée. Ce qui fait que je suis un critique littéraire, mais ce n'est pas ma véritable nature.

C'est cela la vengeance du destin que j'ai fait attendre longtemps - il m'a mis dans une case qui n'est pas exactement le mienne. Ce que j'ai fait - je m'en suis rendu compte très tôt, de cela, que ce n'était pas mon véritable registre. Parce que, aussitôt après avoir commencé à faire de la critique littéraire, j'étais chez un ami, à Ottawa précisément, Ludovic Hudon, que vous connaissez. (Ce n'est pas nécessaire, peut-être, de donner son nom, mais enfin peu importe. Même si son nom est là, cela me fait plaisir.) Il m'a dit: «Je pars faire des courses. Écoutez le quatuor $\mathrm{La}$ Chasse, de Mozart." Alors j'ai mis La Chasse de Mozart sur le pick-up. Et puis, bon, il y avait un bout de papier qui était là, et un stylo-bille. J'ai pris le bout de papier et le stylo-bille et j'ai commencé à écrire Mater Europa, et j'ai écrit jusqu'à la fin. Parce que, à partir de mes articles de critique littéraire, ce petit livre s'était formé en moi et là, 
pfut..., il fallait qu'il sorte. Il est sorti très, très vite, en quinze jours, trois semaines au maximum. Vous voyez, c'était donc une sorte de vengeance du destin.

Mais une demi-vengeance. Je me suis dit: «Puisque essentiellement je suis plutôt un créateur d'imagination qu'un critique, je vais essayer de fondre les deux aspects de ma personnalité." C'est ce qui a été très difficile à accepter. C'est ce que le public a accepté difficilement, surtout les auteurs. C'est-à-dire que mes articles de critique étaient en quelque sorte un miroir dans lequel je me mirais moi-même autant que l'auteur. Et s'il y a quelque chose de déplaisant pour un auteur, c'est cela. C'est de n'être que la moitié de l'article, de ne constituer que la moitié de la présence dans un article de critique. Mais enfin, cela, je ne pouvais pas m'en empêcher puisque c'était mon tempérament.

Vous constatez sans doute, d'ailleurs, que les écrivains, les auteurs de romans et de poèmes ne parlent jamais des autres, ne lisent jamais le livre d'un confrère, cela n'existe pas. Je rencontre des écrivains tout le temps. Je leur parle, je sais bien qu'ils n'ont jamais lu une ligne de moi, jamais, et qu'ils n'en liront jamais une. Cela ne les intéresse pas. Ce qui les intéresse, c'est leur œuvre à eux, ce sont leurs livres. Alors moi je me considère comme quelqu'un qui a fait preuve d'une très grande générosité de tempérament en parlant autant des autres. Personne n'a autant parlé d'écrivains québécois, dans toute l'histoire de la littérature québécoise, que moi. C'est vous dire que je n'ai pas de complexe visà-vis des écrivains québécois. Aucun. Je leur ai rendu plus que ce à quoi ils avaient droit, et de beaucoup.

Je l'ai lancée, d'une certaine façon, cette littérature. Que voulez-vous, en 1960, qui parlait de littérature québécoise? Il y avait Gilles Marcotte, de temps à autre, mais il parlait surtout de livres français qui correspondaient à son école de pensée. Le frère Lockquell, bon, ou il écrivait à 
Québec et il ne parlait pas particulièrement des livres québécois. Ou alors, quand un livre, quand un livre très important paraissait, genre Bonheur d'occasion - c'est un ouvrage charnière, quand même, dans notre littérature -, ou Trente Arpents, des livres qui étaient reconnus à l'étranger, alors on en parlait. Ou alors, Saint-Denys Garneau, on a beaucoup parlé de lui parce qu'il était le chef de file d'une clique qui voulait prendre le pouvoir et qui l'a pris, d'ailleurs, par la suite. Ou alors Hertel, parce que lui aussi avait toute la puissance des jésuites derrière lui. On parlait, comme cela, à titre épisodique, des écrivains québécois. Moi, ce que j'ai fait, c'est que j'en ai parlé de façon continue, et comme d'une littérature qui existait véritablement. J'ai parlé des écrivains québécois comme faisant partie d'une littérature, alors qu'avant on parlait d'eux comme écrivains québécois qui peut-être, un jour, auraient un prix en France. Cela a été quand même pour moi le point de départ d'une écriture. J'ai appris mon métier, en réalité. J'ai appris mon métier d'écrivain. J'ai appris à écrire une phrase française en faisant ce métier de critique littéraire.

M. G. Est-ce que ce travail au Devoir a influencé l'évolution de votre ouvre? Vous avez parlé de Mater Europa. Après Mater Europa, ou à peu près au même moment que Mater Europa, vous avez donné vos deux premiers volumes de Signets. Quand vous êtes passé à la littérature d'imagination, vous avez d'abord commencé par publier des nouvelles, c'est-à-dire des textes courts. Est-ce que cette habitude d'avoir à faire pour le Devoir, toutes les semaines, un texte court a influencé la façon dont votre œuvre s'est développée?

J. É.-B. C'est toujours le même problème qui se pose. C'est l'obstacle à vaincre. Je n'ai pas écrit, au départ, parce que je ne voulais pas me livrer - c'est de cela qu'il s'agit, en fin de compte. J'ai commencé à écrire de la critique parce que je ne voulais pas écrire de nouvelles ou de poésie. Le premier livre que j'ai écrit, Mater Europa, ce 
sont en réalité quatre nouvelles, qui sont reliées entre elles, mais ce sont des nouvelles. Ensuite, j'ai écrit des nouvelles parce que je ne voulais pas écrire un roman. Maintenant, j'ai écrit deux romans, qui sont considérables, en tout cas par le nombre de pages. Donc, quand je m'y suis mis, j’ai rattrapé le temps perdu. Mais je crois que mon véritable registre, c'est le roman. Et c'est ce que je ferai désormais. Je ferai des romans.

\section{LES VOIES DE LA CREATION}

$\boldsymbol{M}$. G. Je crois, effectivement, que vous avez maintenant franchi une étape dans votre parcours d'écrivain. Les Pays étrangers était déjà un roman très réussi, mais c'était un roman qui portait encore sur la matière de votre vie, étant donné que vous y racontez, sous un voile bien transparent, l'histoire de François Hertel et de son apostasie, de la façon dont il a quitté les ordres, et que vous y évoquez également vos souvenirs de collège. Mais cela m'amène à parler de ce roman que vous venez de publier, roman important, Entre toutes les femmes, qui a déjà été très bien reçu. Un peu plus tôt, au cours de cet entretien, vous avez fait allusion à des personnages passionnés qui finissent par tuer. Est-ce que cette œuvre, à vos yeux, marque une étape dans votre carrière, ou un nouveau départ?

I. É. B. Oui. D'abord au point de vue technique. Parce que j'ai compris une grande leçon de l'écriture après avoir écrit ce roman. Ce que j'ai voulu faire, c'est précisément éviter non pas le reproche que vous avez fait en disant: "Les Pays étrangers, c'est encore un reflet de vous-même» mais cela je m'en étais rendu compte. J'ai voulu éviter cette situation un peu fausse, d'écrire des livres qui portent, en réalité, toujours la marque de leur auteur, la marque biographique de leur auteur - d'être témoin, plus qu'un écrivain d'imagination. Alors, je me suis dit: «Le prochain livre que j'écrirai sera un livre aussi objectif que possible, où je n'interviendrai pas du tout.» 
Mais je portais en moi, sans doute, ce personnage d'une petite fille mongolienne. D'ailleurs, un jour, je suis allé dîner chez des gens. On s'est mis à parler d'éducation, etc. Nous n'étions pas du tout du même avis. C'était des gens très comme ça, très collet monté. Il y a une dame qui s'est tournée vers moi et qui m'a dit un peu méchamment: «Mais enfin, Monsieur, est-ce que vous avez des enfants pour parler comme cela de l'éducation des enfants?» Je lui ai dit: «Oui, Madame, je n'ai eu qu'une petite fille et elle est morte à quatorze ans et elle était mongolienne.» Cela a mis fin, naturellement, à ce genre de questions (rires).

Mais c'est vrai. C'est mon enfant, cette petite fille, Anne. Je la portais en moi. Je portais en moi un enfant - est-ce qu'on peut dire monstrueux? non - mais un enfant handicapé ou à part, et qui n'était pas responsable de son état. Je portais cela. Et il fallait que je me délivre de cette strate dans ma nature. Alors, c'est sorti sous la forme d'une petite fille mongolienne et d'un père à la fois très passionné et très ambitieux, du rejet de l'enfant, d'un jeu de dames, en réalité, comme on disait pour la guerre d'Indochine - le damier, où les dames tombent les unes après les autres; elles sont phagocytées les unes par les autres. Donc, j'ai raconté une histoire à partir d'un milieu que je connais, c'est-à-dire le milieu des diplomates, mais cela c'est très superficiel, cela n'a rien à voir, ce pourrait être dans un tout autre milieu. Il y a aussi le milieu des professeurs de médecine - il y a quand même dans ce livre deux professeurs de médecine. Il y a le milieu des intellectuels. Il y a le milieu des étrangers - des réfugiés, si vous voulez - qui viennent habiter le Québec, sous le nom de la princesse Baltykov, etc. Il y a le milieu de cette haute société montréalaise que je connais quand même un peu maintenant.

Tout cela fait que j'ai voulu écrire un livre très objectif. Et je me suis rendu compte, après l'avoir écrit, qu'il n'y a aucun livre qui soit plus subjectif. C'est peut-être le livre où je me livre le plus, beaucoup plus que dans Les Pays étrangers. Je 
suis passé à un autre niveau. Je me suis rendu compte, après avoir écrit ce livre, que plus l'écrivain est objectif, plus il est subjectif, qu'on ne se découvre soi-même, et on ne se révèle et on ne parle bien de soi que lorsqu'on parle des autres, et uniquement des autres, que c'est à ce niveau de profondeur que se situe la révélation de soi. Je me révèle plus en créant un personnage de petite fille mongolienne et en racontant sa mort atroce, qu'en décrivant très longuement le périple intellectuel, psychologique, moral, religieux de HertelGermain Laval, que pourtant je décris de façon très objective. Mais je me révèle plus en parlant de quelque chose que je ne connais absolument pas, sauf par des conversations que j'ai eues avec des médecins sur le mongolisme. Je ne connais aucun enfant mongolien; il n'y en a pas dans ma famille. Pour moi c'était vraiment une terra incognita. Mais cette petite fille est plus que cela. Elle est le symbole de l'amour rejeté, de cet amour que moi je n'ai jamais ressenti. C'est peut-être là la réalité que je veux décrire. C'est essayer de décrire un amour tellement profond qu'il me touchera même moi - il ne m'a pas touché, d'ailleurs. C'est cela qui est terrible, vous comprenez. C'est de sentir très profondément la passion, l'existence de la passion et en même temps de ne pas la ressentir soi-même, et de n'avoir jamais vécu cette passion. Je n'ai jamais aimé mes parents de cette façon, de la façon dont cette petite fille aime. Je n'ai jamais aimé une femme de la façon dont François Bois-le-Duc aime sa femme. Je n'ai jamais été, à l'endroit d'un homme, comme Blanche l'est à l'endroit de son mari. Je n'ai jamais rien ressenti de tout cela. Et pourtant, c'est tout ce que je n'ai pas ressenti qui loge au fond de moi; c'est là, mais à l'état virtuel ou inchoatif. C'est un côté démoniaque, littéralement. C'est un daïmon.

M. G. Est-ce dire que vous vivez à travers vos personnages, par procuration, des choses qui sont inscrites profondément dans votre nature et que vous n'avez pas pu vivre autrement? 


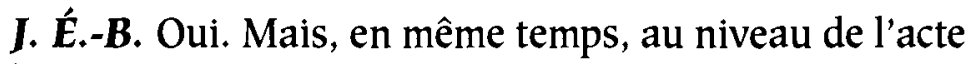
créateur. Je ne les vis pas par l'imagination. Cela ne m'intéresse absolument pas. Je ne peux les vivre que dans la mesure où je leur donnerai la vie. Je ne peux pas, par exemple, me coucher le soir et rêver à des choses comme cela. Pour moi, c'est absolument sans intérêt. Je ne rêve pas à une petite fille mongolienne. Je n'ai jamais rêvé à elle. J'ai pensé à cette enfant la plume à la main. Elle ne vit que dans la mesure où je la crée. Et moi je ne vis cette situation que dans la mesure où cette enfant la crée. Le rapport que j'ai avec des personnages qui vivent ce que moi je ne peux pas vivre, eh bien, ce rapport, je l'ai au niveau de l'imagination créatrice. Et je crois que c'est le cas de tous les romanciers. Mais les romanciers - ceux que je connais, en tout cas - écrivent des choses qu'ils auraient pu vivre. Et moi, ce que je trouve assez curieux, dans mon cas, c'est que je décris et je vis par l'imagination et par la création des sentiments que je n'ai jamais ressentis. Quand vous me disiez, tout à l'heure, l'influence de la lecture sur l'écriture, c'est cela essentiellement. C'est que tout cela relève d'un mariage total avec un univers culturel.

M. G. Tous ces propos, de toute façon, confirment l'une des idées fondamentales de votre ouvre, selon laquelle c'est par l'écriture que l'on s'accomplit.

J. É.-B. Oui, mais enfin quatre-vingt-dix-neuf virgule neuf pour cent des gens ne s'accomplissent pas par l'écriture. Mais c'est par l'écriture que l'écrivain s'accomplit. Moi, c'est mon moyen d'expression. Je n'en ai pas d'autre. C'est mon seul moyen d'expression. Et encore faut-il avoir le désir de s'accomplir. C'est cela, le problème fondamental de l'écrivain. Est-ce que l'écrivain va être comme Michel Déon, qui est un charmant écrivain, qui a du talent, etc., mais est-ce qu'il va rester à un certain niveau de registre? Et moi je crois que l'accomplissement par l'écriture, c'est cela. C'est de passer, par exemple, du niveau — dans mon cas, plutôt - passer du niveau malgré tout anecdotique de la 
critique littéraire avec une sorte de frémissement créateur.

Au niveau de la nouvelle comme, par exemple, ces nouvelles où j'ai ce personnage de Delloise qui revient toujours, cette nouvelle à caractère à la fois mnémotechnique et autobiographique, c'est déjà un pas en avant dans la descente au fond de soi. Passer de cette deuxième étape au roman, comme dans Les Pays étrangers, où la construction du texte intervient de façon très exigeante - vous ne pouvez pas écrire un roman qui ne soit pas construit -, alors il y a l'immense effort intellectuel de construire une œuvre architecturale dans laquelle vous faites entrer des personnages, et qui vivent à l'intérieur de cette œuvre, de cette mécanique - une œuvre architecturale qui est en même temps en mouvement. Vous avez des pavillons entiers qui se déplacent, etc. Vous devez donc avoir une architecture mobile d'une très grande richesse, d'une grande variété, des personnages à l'intérieur de cette architecture mobile et, en plus, qui s'expriment, et faire entrer toute la musique de la vie dans ce mécanisme. Vous avez tous les étages de l'immeuble, les pavillons qui bougent, les personnages qui sont à l'intérieur et qui bougent eux aussi, et tout cela doit créer une sorte de musique cosmique. C'est déjà un grand pas en avant, avec Les Pays étrangers.

Et après, j'en suis venu à Entre toutes les femmes, où le même problème se situe, à mon avis, à un niveau beaucoup plus profond de la réalité psychologique et de la conquête de l'espace psychologique, qui devient en même temps un espace moral sans que ce soit dit, où le psychologique se confond avec le moral. C'est cela, l'accomplissement de l'écrivain. C'est de passer d'une œuvre à l'autre en allant toujours plus loin dans son auto-révélation. C'està-dire que l'écrivain se révèle à lui-même. Je ne crois pas qu'il soit important qu'il se révèle aux autres. Mon destin d'homme n'intéresse personne. Que je me révèle plus ou moins, je ne suis pas la danseuse des sept voiles - comment s'appelle-t-elle? - Salomé... que je me dévoile plus 
ou moins, c'est sans intérêt. C'est pourquoi je trouve que les ouvrages à sensation, de révélation de soi sont complètement idiots, parce que cela n'intéresse que l'auteur. Ce qui intéresse le lecteur, c'est le livre, c'est le résultat. Mais ce qui intéresse l'auteur, c'est l'image qu'il voit de lui-même dans le livre lorsque le livre est terminé. À ce moment-là, il peut savoir s'il est dans un taxi jaune, ou s'il descend au fond d'un puits, à l'intérieur de la terre. C'est une grande différence. Et quand je dis: l'écriture c'est l'accomplissement de soi, c'est ce que je veux dire.

Je vois, par exemple, je viens de terminer un livre de souvenirs d'enfance. J'en ai fait un livre drôle, parce que, en même temps, c'est une œuvre très sérieuse pour moi puisque je raconte mon enfance et des personnages que j'ai connus, et surtout le milieu dans lequel j'ai vécu parce que je ne veux pas laisser se perdre l'image de cette petite ville française, en Ontario, dans les années trente. Je crois que c'est très important au point de vue historique et social. Tous les personnages qui habitaient - pas tous, mais enfin l'organisation de la ville, les rapports entre les Français et les Anglais, je parle de tout cela. Mais un peu sur un mode très légèrement ironique et, en même temps, sentimental et affectueux, etc. Or je me rends compte que j'ai atteint un certain niveau d'écriture, précisément parce que, à la suite d'un livre comme Entre toutes les femmes, je peux écrire l'autre livre, qui est un livre presque d'amusement pour moi, et qui me permet de faire le pont avec le prochain livre qui ne sera pas un livre d'amusement. Ce que je veux faire, désormais, c'est cela, c'est avoir toujours une œuvre importante pour moi en train, comme ce livre que je veux écrire sur un chef d'orchestre, la vie d'un chef d'orchestre, qui sera un livre, en réalité, consacré aux rapports entre... un livre consacré au thème de Job, dans la Bible, l'homme qui a tout, qui perd tout, et qui, à la fin, retrouve tout amélioré. Je veux, d'une part, écrire ce genre de livre et, en même temps, avoir à côté l'œuvre, non pas de circonstance, mais l'œuvre 
d'ouverture et d'atmosphère poétique.

$\boldsymbol{M}$. G. De sorte que vous êtes un écrivain qui est encore en pleine activité créatrice.

J. $\boldsymbol{E}$.-B. Je suis en période de gestation. C'est-à-dire que je ne fais que commencer à écrire mon œuvre. Je commence à écrire une œuvre à l'âge où les autres détellent. À soixante ans, d'habitude, les écrivains disent: «Bon, maintenant je vais écrire des choses comme ça, un article de temps à autre.» Les écrivains de ma génération n'écrivent plus. C'est fini. Alors que moi, j'ai très bien fait, en réalité, d'attendre et d'engranger des expériences humaines pendant des années et des années et des années. Et tout cela est allé dans des petits modules à l'intérieur du cerveau. Et maintenant je n'ai véritablement qu'à appuyer sur le bouton et il y a quelque chose qui sort, et qui correspond exactement à ce que je veux dans le livre. Le geste qu'une femme fait dans un livre, ou un homme, dans une chose que j'écris, je l'ai vu... quelque part, je ne sais pas où. C'est peut-être un guide - nous allons faire de la culture dans la forêt de Siem Reap, alors que j'étais en train de visiter les temples d'Angkor, qui a fait un geste, et ce geste je l'ai noté et il ressort longtemps après. Le décor, même. Dans Entre toutes les femmes, en haut de l'escalier, il y a une bibliothèque qui est là et qui est éclairée. Cette bibliothèque, je la connais. Les livres qu'il y a dedans ne sont pas les mêmes. Le personnage de Don Quichotte, qui est un personnage qui me hante, eh bien, il apparaît; il faut qu'il apparaisse. Mes livres, en réalité, sont des livres peuplés de vieux amis, qui sont là sous une forme ou sous une autre, et qui sont transposés, qui sont transformés, qui sont recréés...

M. G. ... mais que vous devez sans doute voir très clairement en vous pour être capable...

J. E. . - B. ... après, je les revois très clairement...

M. G. ... après coup... 
J. É.-B. ... oui. Pendant, je ne les vois pas. Ils s'imposent à moi. Un exemple - je ne sais pas si j'ai parlé de cela à Gérard-Marie Boivin, mais c'est un exemple très convaincant. J'étais en train d'écrire Entre toutes les femmes. J'ai dû l'abandonner deux fois, parce que les scènes que je voulais écrire étaient trop violentes pour moi. Alors il a fallu que je les laisse reposer, bon, et je les écris. Mais, à un moment donné, le personnage de Blanche ne réagissait pas du tout. Elle n'existait plus. Je ne m'entendais pas avec elle. Je ne pouvais pas la faire bouger. Elle était butée - c'était comme une sorte d'ânesse, dans un coin, et qui refuse de bouger. Je me suis dit: «Tant pis, je ferai autre chose, et ce livre je ne veux plus en entendre parler.» Il me sortait par les trous du nez - parce qu'il m'a donné beaucoup de difficultés psychologiques. Je l'ai mis carrément dans un tiroir. Et, un jour, peut-être trois ou quatre mois après, tout d'un coup je me suis dit: «Mais je la reprends. Je vais voir si elle s'est un peu calmée.» Elle s'était calmée. Elle est revenue dans le livre, mais elle avait emmené avec elle une amie - c'est la princesse Baltykov, qui arrive dans l'histoire à peu près aux deux tiers du livre. Elle n'est pas du tout présente au début. Elle arrive à la fin, pourquoi? Parce que, pour mener cette histoire à bien, Blanche $\mathrm{a}$ besoin d'un appui. Elle est revenue. Elle a emmené avec elle quelqu'un qui est un témoin, qui est une conseillère, qui est un appui, qui est sa seule amie - la seule personne en qui elle ait véritablement confiance. Elle est allée la chercher, elle, à l'extérieur du livre, elle l'a emmenée et j'ai été obligé d'accepter la présence de la princesse Baltykov.

Vous voyez comme c'est curieux, le rapport avec le personnage. Il faut vivre avec cela. Il faut apprendre à accepter avec beaucoup de modestie, en réalité, que le personnage lui-même soit à ce point autonome, qu'il agisse contre vous et qu'il règle les problèmes que vous avez. Et qu'il s'impose. Il ne faut pas malmener les personnages. Il faut les laisser libres, [les laisser] agir, et ils font comme ils veulent. Voyez 
François, qui est un personnage dur et, d'une certaine façon, assez mesquin. Eh bien, malgré tout, j'ai une certaine tendresse pour lui. Alors, je lui ai fait un beau cadeau, à la fin. Quand il meurt, il meurt avec Don Quichotte. On ne peut pas faire un plus beau cadeau à un homme que celui-là..., de le faire mourir en pensant à Don Quichotte et à Dulcinée.

$\boldsymbol{M}$. G. Je trouve le titre de ce roman fort bien choisi, parce que, d'abord, il me semble résumer très bien l'espèce d'élection de Blanche, "entre toutes les femmes", pour accomplir un destin, mais également parce qu'il me semble que les personnages vraiment forts de ce livre - comme tendent à le confirmer, d'ailleurs, les propos que vous teniez à l'instant - les personnages vraiment importants de ce livre sont des femmes. Il y a Blanche, il y a Anne, il y a Madame Bois-le-Duc mère. Et j'avais déjà noté la même chose dans Les Pays étrangers. Madame Soublière et sa fille sont des personnages très forts et très attachants. Estce que cela a une certaine importance pour vous, le fait d'avoir réussi à produire des personnages féminins qui sont des personnages très forts?

J. É.-B. Je crois que mon intelligence et ma volonté sont d'un homme, et ma sensibilité très féminine.

M. G. À quoi cela tient-il?

J. É.-B. À ma nature... Je suis comme cela, et je l'accepte d'ailleurs. Et c'est ce qui me permet, d'instinct - d'abord, je suis en dehors de la femme, donc j'ai une sorte d'objectivité au départ - mais mon instinct me porte vers la compréhension de la femme, tout en restant en dehors et en restant objectif. C'est ce qui fait, je crois, que les personnages de femmes sont, si vous voulez, plus réussis que les personnages d'hommes. Les personnages d'hommes que je réussis sont des personnages épisodiques. Par exemple, le professeur Bois-le-Duc. Je pense que c'est un personnage réussi, parce qu'il est un peu à l'écart, il a une personnalité à lui, et il meurt d'une façon très agréable. Lui, il 
existe vraiment. L'homme, je n'aime pas. C'est très difficile, pour moi, de créer des personnages d'hommes avec amour puisque je ne les aime pas. C'est la femme que j'aime, en réalité. Mais, en même temps, de façon très objective. Je vais vers elle du dehors, mais avec ma sensibilité qui frémit au même rythme que la sienne.

\section{L'ACTION}

M. G. Si vous le permettez, je voudrais passer maintenant à un autre aspect de votre activité. Parce qu'à côté de votre travail solitaire d'écrivain créateur, vous avez également été très présent sur la place publique, dans les débats de société que nous avons connus ces dernières années. Vous avez été un écrivain au service de l'écriture et des écrivains. J'en vois la preuve dans ce congrès mondial du PEN Club que vous organisez pour le mois de septembre de cette année [1989]. Est-ce que vous pourriez nous parler un peu de votre engagement de ce côté-là, nous dire ce que représente votre travail au PEN et ce que peut signifier ce congrès pour la littérature d'ici?

I. É.-B. D'abord, je vais parler un peu du PEN Club, qui est une organisation mondiale d'écrivains, d'entraide, en réalité, et qui a soixante-douze centres autonomes dans le monde entier, et qui s'occupe, plus particulièrement, d'aider les écrivains en prison. C'est le but, en ce moment, le plus important du PEN Club. Nous-mêmes, le Centre francophone canadien, nous avons beaucoup œuvré en ce sens. Le PEN Club canadien faisait la même chose.

Il y a cinq ans, on m'a élu président de ce PEN Club canadien. Le siège social était à Montréal. Et je me suis rendu compte qu'en réalité, il valait peut-être mieux, il aurait peut-être mieux valu que le PEN Club se scinde et que nous formions un PEN Club d'écrivains francophones. C'est pourquoi le Centre francophone canadien a été fondé: pour permettre aux écrivains de langue française, du Québec 
surtout, bien sûr, et du reste du Canada - le Québec et le Centre francophone canadien étant responsables des écrivains de langue française à l'extérieur du Québec -, de s'affirmer comme entité autonome au niveau international. Donc j'ai fondé le PEN Club francophone canadien.

Cette année, de conserve avec nos confrères de Toronto, nous organisons ce congrès mondial - trois jours à Toronto, trois jours à Montréal.

Vous parlez d'engagement dans la société. Je crois qu'il est essentiel pour tous les écrivains, à un moment ou à un autre dans leur vie, de se manifester en tant qu'écrivains et d'assumer la responsabilité de leurs confrères. C'est un acte d'écriture, qui est aussi important que d'écrire un livre. Cet engagement veut peut-être dire que cet écrivain écrira un livre de moins dans sa bibliographie. "Du même auteur' n'aura pas ce titre, mais il aura eu droit à cet enrichissement de participation très profonde à la vie mondiale des écrivains, ou à la vie municipale des écrivains, ou à la vie provinciale, nationale, peu importe. Mais je crois qu'il faut, une fois dans la vie, cet engagement. Moi, je le fais maintenant. Après le congrès mondial du PEN Club, j'aurai fait mon devoir. J'estimerai que j'ai le droit très strict de retourner à mes travaux et de faire mon œuvre d'écrivain sans plus me préoccuper des autres, sauf peutêtre dans le cas des écrivains en prison où là, naturellement, l'être humain en soi réagit aussi devant cette situation. Ce n'est pas seulement une réaction d'écrivain, mais une situation d'homme.

Nous organisons donc ce congrès pour la fin de septembre. Nous serons environ... - en ce moment nous sommes en train d'organiser le banquet de clôture et nous attendons environ sept cents personnes. C'est un congrès qui sera accompagné de beaucoup d'activités adventices, comme par exemple un très grand lancement de livres québécois, un festival du film d'auteurs. Nous avons commandé une 
grande cantate sur le thème de l'écrivain et de la paix. Nous avons confié le soin d'écrire cette œuvre à Michel Longtin, qui est un musicien très organisé, très sérieux, et jeune en plus - c'est aussi un peu pourquoi nous l'avons choisi, mais surtout, il va de soi, à cause de son grand talent. Dans l'ensemble, notre organisation marche très bien.

Cette organisation m'a permis de comprendre le mécanisme aussi de la quête des fonds dans notre société. J'ai trouvé cela très intéressant que de rencontrer des hommes d'affaires, des magnats de la presse, de l'industrie et de discuter avec eux de l'organisation de ce congrès et d'essayer de les convaincre de participer à nos activités. Cela m'a beaucoup, beaucoup intéressé. Cela m'a ouvert une porte sur un monde que j'ignorais et que je ne fréquenterai jamais, mais que j'ai trouvé intéressant parce que j'ai vu les mécanismes de décision à l'œuvre. Je me suis dit: «Eh bien voilà un volet du monde dans lequel nous vivons, que je ne connaissais pas et que maintenant je comprendrai mieux." Et je me suis trouvé en face de gens souvent très intelligents et de véritables mécènes. Un type comme Marcel Couture, par exemple, d'Hydro-Québec, est un homme dont le tempérament est un tempérament de mécène. Et j'ai vu aussi que ces grandes sociétés jouent un rôle très important dans notre vie culturelle. Une maison comme Lavalin, par exemple, est un élément charnière de la vie québécoise, et canadienne j'imagine. Et HydroQuébec est très remarquable. Par exemple, Hydro-Québec et Alcan donneront désormais tous les ans chacun dix mille dollars - c'est-à-dire, ensemble, vingt mille dollars au PEN Club, à notre Centre, pour donner un prix à un écrivain de la francophonie. C'est admirable, quand on y pense. Vous me direz: "Il y a des pannes d'électricité." Mais il n'y a pas de pannes d'électricité parce que le PEN Club décernera un prix de vingt mille dollars par année. Il y a des pannes d'électricité parce qu'on ne donne pas 
cinquante mille. Si on avait donné cinquante mille, il n'y aurait pas eu de panne (rires).

Nous attendons des écrivains de très grand renom dans toutes les langues du monde. Nous aurons des gens comme Czesłkaw Milosz ${ }^{7}$, le prix Nobel. Et, chez les Québécois, naturellement, les écrivains les plus importants participeront à notre congrès.

M. G. De sorte que vous voyez ce congrès comme un stimulant puissant pour notre monde littéraire.

J. $\dot{\boldsymbol{E}}$. B. Très puissant. Et je dois ajouter (cela j'aimerais que vous le mettiez dans le texte) que nous faisons notre congrès dans l'harmonie la plus étroite avec les autres sociétés littéraires les plus importantes, comme, par exemple, la Société des écrivains (dont je suis le président, naturellement $-c$ 'est dans la plus grande harmonie, comme vous voyez [rires]), mais aussi que nous avons la collaboration la plus étroite avec l'Union des écrivains québécois et son président, Bruno Roy, qui est un homme d'un grand sérieux, excellent organisateur, et qui a le plus profond respect, comme il se doit, de la chose littéraire. C'est très intéressant pour nous de constater qu'il y a autour de ce congrès une unité de pensée chez les écrivains québécois et au sein des organisations qui les représentent.

M. G. Que vous réserve l'avenir, Jean Éthier-Blais, audelà de ce congrès?

J. É. -B. D'abord, je suis presque en fin de carrière. J'ai l'intention de prendre ma retraite bientôt, le plus tôt possible, et d'écrire, et de consacrer ma vie à répondre à ce besoin.

M. G. Vous avez dit quelque part, je ne sais plus où, que vous faites une sorte de contrat avec le temps, de façon à pouvoir poursuivre votre œuvre. C'est ce qui confirme que vous êtes encore en pleine création, en pleine ébullition

${ }^{7}$ Czesłkaw Milosz ne figura pas, en fin de compte, au programme de ces assises. 
créatrice, et sans doute pouvons-nous attendre de vous encore un grand nombre d'œuvres...

J. É.-B. Je le souhaite, surtout pour moi. Pour vous, c'est autre chose: tout dépendra de la qualité des œuvres. Mais, pour moi, je pense que ce serait un supplément de bonheur dans la vie que de pouvoir continuer à écrire comme je le fais en ce moment.

M. G. Jean Éthier-Blais, il ne me reste plus qu'à vous remercier de vous être prêté de si bonne grâce à cette interview et d'avoir accepté de partager avec nous les choses si profondes que vous venez de dire sur votre œuvre et sur votre métier d'écrivain. 